\title{
THE PERPENDICULAR NEUMANN PROBLEM FOR MEAN CURVATURE FLOW WITH A TIMELIKE CONE BOUNDARY CONDITION
}

\author{
BEN LAMBERT
}

\begin{abstract}
This paper demonstrates existence for all time of mean curvature flow in Minkowski space with a perpendicular Neumann boundary condition, where the boundary manifold is a convex cone and the flowing manifold is initially spacelike. Using a blowdown argument, we show that under renormalisation this flow converges towards a homothetically expanding hyperbolic solution.
\end{abstract}

\section{INTRODUCTION}

In this paper we work with Mean Curvature Flow (MCF) of hypersurfaces in Minkowski space with a perpendicular Neumann boundary condition. We show that if the boundary manifold is a convex cone made up of timelike rays, then MCF will have a solution for all time for any initial spacelike hypersurface satisfying the boundary condition. If we renormalise to keep volume of the flowing manifold constant, then we see that this hypersurface converges to a homothetic solution to MCF, specifically that given by flowing a hyperbolic hyperplane (see Definition 1.2). Since these hyperbolic hyperplanes are sets of points of constant negative "radius" in Minkowski space, this may be seen as a Minkowski-Neumann analogue of 7 .

MCF with a perpendicular Neumann condition in the Euclidean setting was considered as a graph over $C^{2+\alpha}$ domains by Huisken in [8], where a longtime existence and convergence result was obtained. Altschuler and Wu [1] also considered graphs with a varying angle Neumann condition, although only with flowing surfaces of dimension 2 and over convex domains, yielding convergence to translating solutions. In [10] and [11, Stahl dealt with perpendicular boundary conditions but this time not necessarily for graphs. He showed that when the boundary is an umbilic hypersurface, a convex initial surface moving by MCF converges to a round point. Buckland [2] found boundary monotonicity formulae for MCF and classified Type I boundary singularities for $H>0$ with a perpendicular Neumann boundary condition.

MCF (and related flows) have been used in Minkowski space by Ecker and Huisken in [5] to construct entire surfaces of constant mean curvature. In [3], Ecker dealt with both the Dirichlet boundary condition and with the entire case, showing that without any growth conditions on the initial data, the entire flow exists for all time. He also showed that for any graphical spacelike initial data MCF with a Dirichlet boundary condition, the flow exists for all time and converges to

Received by the editors December 8, 2011.

2010 Mathematics Subject Classification. Primary 53C44, 35K59.

(c) 2014 American Mathematical Society Reverts to public domain 28 years from publication 
a maximal surface. In [4, he generalised this to the degenerate Dirichlet boundary case in more general semi-Riemannian manifolds. For a recent application of spacelike MCF see the work of Guilfoyle and Klingenberg [6].

1.1. Definitions and notation. We will need several definitions: Define $\mathbb{R}_{1}^{n+1}$ (where $n \geq 2$ ) to be Minkowski space that is $\mathbb{R}^{n+1}$ equipped with the indefinite metric $\langle-,-\rangle$ where

$$
\langle\mathbf{x}, \mathbf{y}\rangle=x_{1} y_{1}+\ldots+x_{n} y_{n}-x_{n+1} y_{n+1} .
$$

Let $\Sigma$ be a smooth embedded hypersurface in $\mathbb{R}_{1}^{n+1}$ with an indefinite metric, which is to say it is possible to find locally $n$ orthogonal vector fields $X_{i}$ for $1 \leq i \leq n-1$ and $Y$ such that $\left\langle X_{i}, X_{i}\right\rangle=1$ and $\langle Y, Y\rangle=-1$. This will be the boundary manifold, and let $\mu$ be its outward pointing unit normal. Our intention is to flow $M^{n}$, a smooth $n$-dimensional topological manifold with boundary $\partial M$, from an initial spacelike embedding $\mathbf{F}_{0}: M^{n} \rightarrow \mathbb{R}_{1}^{n+1}$. We specify additionally that $\mathbf{F}_{0}(\partial M) \subset \Sigma$ with the extra compatibility condition $\langle\nu, \mu\rangle=0$, where $\nu$ is the normal to $M_{0}=\mathbf{F}_{0}\left(M^{n}\right)$.

Definition 1.1. Let $\mathbf{F}: M^{n} \times[0, T] \rightarrow \mathbb{R}_{1}^{n+1}$ be such that

$$
\begin{cases}\frac{d \mathbf{F}}{d t}=\mathbf{H}=H \nu & \forall(x, t) \in M^{n} \times[0, T], \\ \mathbf{F}(\cdot, 0)=M_{0}, & \\ \mathbf{F}(x, t) \subset \Sigma & \forall(x, t) \in \partial M^{n} \times[0, T], \\ \langle\nu, \mu\rangle(x, t)=0 & \forall(x, t) \in \partial M^{n} \times[0, T] .\end{cases}
$$

Then $\mathbf{F}$ moves by Mean Curvature Flow with a Neumann free boundary condition $\Sigma$ (here $\nu(x, t)$ is the normal to $\mathbf{F}$ at time $t$ ).

We will need various geometric quantities on various manifolds. A bar will imply quantities on $\mathbb{R}_{1}^{n+1}$, for example $\bar{\Delta}, \bar{\nabla}, \ldots$ and so on; no extra markings $\Delta, \nabla, \ldots$ will refer to geometric quantities on $M_{t}$, our flowing surface at time $t$, and for any other manifold $Z, \Delta^{Z}, \nabla^{Z}, \ldots$, etc. will refer to the Laplacian, covariant derivatives, ... on $Z$.

In this paper we will choose $\Sigma$ to be a timelike cone - a cone in Minkowski space centred at $\mathbf{0}$ such that at any point except $\mathbf{0}$, the tangent space has a strictly timelike vector (see below for full details). Such a cone divides the half space $\left\{\mathbf{x} \in \mathbb{R}_{1}^{n+1} \mid x_{n+1}>0\right\}$ into two components. We define the interior of $\Sigma$ to be the component such that every position vector is timelike.

In Minkowski space we have the equivalent of the homothetically shrinking sphere - the homothetically expanding hyperbolic hyperplane.

Definition 1.2. We define the expanding hyperbolic hyperplane $\mathbf{G}_{k}$ for $k>0$ to be the solution to (1), starting with the hyperbolic hyperplane of "radius" $k$ intersected with the interior of $\Sigma$. That is at time $t=0,\left\langle\mathbf{G}_{k}, \mathbf{G}_{k}\right\rangle=-k^{2}$ with $\left(\mathbf{G}_{k}\right)_{n+1}>0$. It is easy to show that the boundary conditions are satisfied and that

$$
-\left\langle\mathbf{G}_{k}, \mathbf{G}_{k}\right\rangle=k^{2}+2 n t .
$$

In this paper we first obtain the following longtime existence result:

Theorem 1.3. Let $\Sigma$ be a convex cone. Given that $M_{0}$ is initially spacelike, then a solution to equation (1) exists for all time. Furthermore, this solution stays between two homothetic solutions $\mathbf{G}_{C_{0}}$ and $\mathbf{G}_{C_{1}}$, where $C_{0}$ and $C_{1}$ are the minimum and maximum values of $\sqrt{-\langle\mathbf{F}, \mathbf{F}\rangle}$ at time $t=0$. 
We are able to get further convergence results. We define $\widehat{M}$ to be the blowdown of $M$, that is, $M$ renormalised by dilations so that $\widehat{M}$ has constant unit area. By defining convergence "at infinity" to be the convergence of $\widehat{M}$, we get the following:

Theorem 1.4. Any initially spacelike solution of equation (1) with a convex cone boundary condition under renormalisation will converge to a homothetic solution in the $C^{1}$ norm. Furthermore, there exists an increasing sequence of $t_{i} \rightarrow \infty$ such that the $\widehat{M}_{t_{i}}$ converge to the solution in the interior in the $C^{\infty}$ topology.

\section{A REPARAMETRISATION}

For simplicity we may reparametrise the above system as a graph over a topological disc $D \subset B_{1}^{n}(\mathbf{0})$ defined by the intersection of the interior of $\Sigma$ with the hyperplane perpendicular to $\mathbf{e}_{n+1}$ and intersecting $(0, \ldots, 0,1)$. We may then describe a spacelike manifold $M$ inside $\Sigma$ as follows: At a point $\mathbf{x} \in D$ if we take the ray from $\mathbf{0}$ through $\mathbf{x}$, then the ray will intersect $M$ only once. If $\mathbf{p}$ is that point of intersection, then let $u(\mathbf{x})=\sqrt{-\langle\mathbf{p}, \mathbf{p}\rangle}$. The graph $u$ now parametrises $M$ by $\mathbf{F}(x)=u(x) \frac{\mathbf{x}+\mathbf{e}_{n+1}}{\sqrt{1-|\mathbf{x}|^{2}}}$. Standard calculations give geometric quantities; for example,

$$
g_{i j}=\frac{u^{2}}{1-|\mathbf{x}|^{2}}\left(\delta_{i j}+\frac{x_{i} x_{j}}{1-|\mathbf{x}|^{2}}\right)-D_{i} u D_{j} u
$$

and

$$
\nu=\frac{\left(1-|\mathbf{x}|^{2}\right) \mathbf{D} u+u \mathbf{x}+\left(D u \cdot x\left(1-|\mathbf{x}|^{2}\right)+u\right) \mathbf{e}_{n+1}}{\left(1-|\mathbf{x}|^{2}\right) v},
$$

where $v$ is a gradientlike function

$$
v=\sqrt{\frac{u^{2}}{1-|\mathbf{x}|^{2}}+(D u \cdot \mathbf{x})^{2}-|D u|^{2}} .
$$

By this method we see that a solution to $\mathrm{MCF}$ is equivalent to a solution to the following parabolic quasilinear PDE: For $u: D \times[0, T) \rightarrow \mathbb{R}$

$$
\begin{cases}\frac{d u}{d t}=\frac{v H \sqrt{1-|\mathbf{x}|^{2}}}{u}=g^{i j} D_{i j} u+\frac{n+1}{u}-\frac{1}{v^{2}}\left(\frac{u}{1-|\mathbf{x}|^{2}}+2 D u . \mathbf{x}\right) & \forall \mathbf{x} \in D, \\ u(\mathbf{x}, 0)=u_{0}(\mathbf{x}) & \forall \mathbf{x} \in D, \\ D u \cdot(\gamma-\gamma \cdot x \mathbf{x})=0 & \forall \mathbf{x} \in \partial D\end{cases}
$$

holds, where

$$
\begin{aligned}
g^{i j}=\frac{1-|\mathbf{x}|^{2}}{u^{2}}\left(\delta_{i j}+\frac{1}{v^{2}}\right. & {\left[\left(|D u|^{2}-\frac{u^{2}}{1-|\mathbf{x}|^{2}}\right) x_{i} x_{j}\right.} \\
& \left.\left.+D_{i} u D_{j} u-D u \cdot \mathbf{x}\left(x_{i} D_{j} u+x_{j} D_{i} u\right)\right]\right)
\end{aligned}
$$

is the inverse of the metric and $\gamma$ is the outward pointing unit normal to $D$. Longterm existence is equivalent to uniform parabolicity of the above equation and $C^{1}$ bounds on $u$. By calculating eigenvalues of the metric, $g_{i j}$, we see that this is equivalent to bounding $\max \left\{\frac{1}{v^{2}}, \frac{1}{u^{2}}, u^{2}\right\}$ from above. 


\section{The Boundary MANifold}

Here we will define more rigorously the boundary manifold $\Sigma$ and state formulae for its curvature. Let $\widetilde{\mathbf{S}}: S^{n} \rightarrow B_{1}(0) \subset \mathbb{R}^{n}$ be a smooth embedding of a sphere into the unit ball centred at the origin with outward unit normal $\mathbf{n}$. Then we may define a boundary cone $\Sigma_{\widetilde{\mathbf{S}}}$ (later the subscript will be dropped) by embedding $\mathbb{R}^{n}$ into $\mathbb{R}_{1}^{n+1}$ at height 1 and then defining $\Sigma_{\widetilde{\mathbf{S}}}$ to be the set of all rays going through the origin and some point $(\widetilde{\mathbf{S}}(x), 1)$. More explicitly we may give a parametrisation $\mathbf{S}:(0, \infty) \times S^{n} \rightarrow \mathbb{R}_{1}^{n+1}$ of $\Sigma_{\widetilde{\mathbf{S}}}$ by

$$
(l, x) \mapsto l \widetilde{\mathbf{S}}(x)+l \mathbf{e}_{n+1} .
$$

We may now calculate all quantities needed. For example, we may now see that in these coordinates

$$
\begin{gathered}
A^{\Sigma}\left(\cdot, \frac{\partial}{\partial l}\right)=0 \\
A^{\Sigma}\left(\frac{\partial}{\partial x^{i}}, \frac{\partial}{\partial x^{j}}\right)=\frac{l A^{\widetilde{S}}\left(\frac{\partial}{\partial x^{i}}, \frac{\partial}{\partial x^{j}}\right)}{\sqrt{1-\langle\widetilde{\mathbf{S}}, \mathbf{n}\rangle^{2}}} .
\end{gathered}
$$

Therefore, for an orthonormal set of vectors $\mathbf{e}_{l}, \mathbf{e}_{1}, \ldots, \mathbf{e}_{n-1} \in T_{\mathbf{p}} \Sigma$ obtained by picking orthonormal coordinates on $\widetilde{S}$ and renormalising, then

$$
A^{\Sigma}\left(\mathbf{e}_{i}, \mathbf{e}_{j}\right)=\frac{A^{\widetilde{S}}\left(\mathbf{e}_{i}, \mathbf{e}_{j}\right)}{l \sqrt{1-\langle\widetilde{\mathbf{S}}, \mathbf{n}\rangle^{2}}} .
$$

Hence we can see that, as we would expect, convexity of $\Sigma$ is equivalent to convexity of the embedding $\widetilde{\mathbf{S}}$, the second fundamental form has a zero eigenvector along the timelike rays from the origin, and the second fundamental form decreases linearly as you move up the cone.

\section{Evolution equations}

In this section we will derive some useful evolution equations by straightforward calculation. We define the following:

$$
\begin{gathered}
F^{2}=-\langle\mathbf{F}, \mathbf{F}\rangle>0, \\
S=-\langle\mathbf{F}, \nu\rangle>F .
\end{gathered}
$$

We may think of these as in some sense $C^{0}$ and $C^{1}$ measures of how far our flowing manifold is from a homothetic solution $\mathbf{G}_{k}$.

Lemma 4.1. Under $M C F$ we have

$$
\left(\frac{d}{d t}-\Delta\right) F^{2}=2 n
$$

Proof. We have

and

$$
\frac{d F^{2}}{d t}=-2 H\langle\nu, \mathbf{F}\rangle=2 H S
$$

$$
\begin{aligned}
\Delta F^{2} & =-2 g^{i j}\left(h_{i j}\langle\nu, \mathbf{F}\rangle+g_{i j}\right) \\
& =2 H S-2 n .
\end{aligned}
$$


Lemma 4.2. The normal $\nu$ evolves by

$$
\frac{d \nu}{d t}=\nabla H
$$

Proof. See for example [5. Proposition 3.1].

Lemma 4.3. In the interior of the flowing manifold we have

$$
\left(\frac{d}{d t}-\Delta\right) S=2 H-S|A|^{2}
$$

Proof. Using Lemma 4.2 we get

$$
\frac{d S}{d t}=H-\left\langle\mathbf{F}^{\top}, \nabla H\right\rangle
$$

and

$$
\begin{aligned}
\Delta S & =-g^{i j}\left(\nabla_{\frac{\partial}{\partial x^{i}}} A\left(\mathbf{F}^{\top}, \frac{\partial}{\partial x^{j}}\right)+A\left(\nabla_{\frac{\partial}{\partial x^{i}}} \mathbf{F}^{\top}, \frac{\partial}{\partial x^{j}}\right)\right) \\
& =-\nabla_{\mathbf{F}^{\top}} H-g^{i j} A\left(\nabla_{\frac{\partial}{\partial x^{i}}} \mathbf{F}^{\top}, \frac{\partial}{\partial x^{j}}\right) .
\end{aligned}
$$

Now we calculate

$$
\begin{aligned}
g^{i j} A\left(\nabla_{\frac{\partial}{\partial x^{i}}} \mathbf{F}^{\top}, \frac{\partial}{\partial x^{j}}\right) & =g^{i j} A\left(\left(\bar{\nabla}_{\frac{\partial}{\partial x^{i}}}(\mathbf{F}-S \nu)\right)^{\top}, \frac{\partial}{\partial x^{j}}\right) \\
& =H-S|A|^{2}
\end{aligned}
$$

which shows

$$
\Delta S=-\nabla_{\mathbf{F}^{\top}} H-H+S|A|^{2},
$$

and we have the lemma.

We also need several evolution equations for the curvature.

Proposition 4.4. In the interior of the flowing manifold we have for $m \geq 1$

$$
\begin{aligned}
& \left(\frac{d}{d t}-\Delta\right) H=-H|A|^{2} \\
& \left(\frac{d}{d t}-\Delta\right)|A|^{2}=-2|A|^{4}-2|\nabla A|^{2}, \\
& \left(\frac{d}{d t}-\Delta\right)\left|\nabla^{m} A\right|^{2} \leq-2\left|\nabla^{m+1} A\right|^{2} \\
& +\sum_{i+j+k=m} \nabla^{i} A * \nabla^{j} A * \nabla^{k} A * \nabla^{m} A .
\end{aligned}
$$

Proof. See [5, Proposition 3.3].

\section{Boundary DERIVATIVES}

To apply the Hopf maximum principle we also need to consider derivatives of functions at the boundary in the direction of $\mu$, the normal to $\Sigma$. As in the case of Stahl [10], these identities come from derivatives of the boundary condition. We first demonstrate the following simple result.

Lemma 5.1. At a point $p \in \partial M^{n} \times[0, T)$ we have

$$
\left\langle\nabla F^{2}, \mu\right\rangle=0 .
$$


Proof. We have that $\nabla F^{2}=\left(\bar{\nabla} F^{2}\right)^{\top}$. Since $\bar{\nabla} F^{2} \in T_{p} \Sigma$ we see, using the boundary condition, that

$$
\left\langle\mu, \nabla F^{2}\right\rangle=\left\langle\mu, \bar{\nabla} F^{2}+\left\langle\nu, \nabla F^{2}\right\rangle \nu\right\rangle=0
$$

Now we take spatial derivatives of the boundary condition to give:

Lemma 5.2. For $W \in T_{p} M_{t} \cap T_{p} \Sigma$, then

$$
A(\mu, W)=-A^{\Sigma}(\nu, W) \text {. }
$$

Proof.

$$
0=W\langle\nu, \mu\rangle_{\mathbb{R}_{1}^{n+1}}=\left\langle\bar{\nabla}_{W} \nu, \mu\right\rangle+\left\langle\nu, \bar{\nabla}_{W} \mu\right\rangle=A(W, \mu)+A^{\Sigma}(\nu, W) .
$$

For our gradient estimate we also need

Lemma 5.3. For $p \in \partial M^{n} \times[0, T)$ we have

$$
\langle\nabla S, \mu\rangle=-S A^{\Sigma}(\nu, \nu) .
$$

Proof.

$$
\nabla S=-\left\langle\mathbf{F}, \frac{\partial \nu}{\partial x^{i}}\right\rangle g^{i j} \frac{\partial}{\partial x^{j}}=-A\left(\mathbf{F}^{\top}, \frac{\partial}{\partial x^{i}}\right) g^{i j} \frac{\partial}{\partial x^{j}},
$$

and so taking an inner product and applying Lemma 5.2 ,

$$
\langle\nabla S, \mu\rangle=-A\left(F^{\top}, \mu\right)=A^{\Sigma}\left(F^{\top}, \nu\right) .
$$

Using the fact that the second fundamental form of $\Sigma$ has a zero eigenvector in the direction $\mathbf{F}$, then

$$
A^{\Sigma}\left(F^{\top}, \nu\right)=A^{\Sigma}(\mathbf{F}, \nu)-S A^{\Sigma}(\nu, \nu)=-S A^{\Sigma}(\nu, \nu) .
$$

Now differentiating the boundary condition with respect to time:

Lemma 5.4. For $p \in \partial M^{n} \times[0, T)$ we have

$$
\langle\nabla H, \mu\rangle=-H A^{\Sigma}(\nu, \nu) \text {. }
$$

Proof. Using Lemma 4.2

$$
0=\frac{d}{d t}\left\langle\nu, \mu_{\left.\right|_{\mathbf{F}}}\right\rangle_{\mathbb{R}_{1}^{n+1}}=\langle\nabla H, \mu\rangle+\langle\nu, D \mu(H \nu)\rangle_{\mathbb{R}_{1}^{n+1}}=\langle\nabla H, \mu\rangle+H A^{\Sigma}(\nu, \nu) .
$$

Remark 5.5. We note that if $\Sigma$ is convex, then the normal derivatives at the boundary of both $H$ and $S$ are negative.

On the other hand, regardless of the boundary, we are able to get

Corollary 5.6. For $p \in \partial M^{n} \times[0, T)$ we have

$$
\left\langle\nabla \frac{H}{S}, \mu\right\rangle=0
$$




\section{Gradient estimate}

We now obtain a gradient estimate, that is to say, a lower bound on $v$. Note that in the graphical notation of equation (2)

$$
S=\frac{u^{2}}{v \sqrt{1-|\mathbf{x}|^{2}}} .
$$

Hence for longtime existence it is sufficient to find a suitable upper bound on $S$ and upper and lower bounds on $u^{2}=F^{2}$. We will need an assumption:

Assumption 6.1. We will assume from here on that $\Sigma$ is convex.

We also require a maximum principle:

Theorem 6.2 (Weak Maximum Principle). Suppose we have a function $f: M^{n} \times$ $[0, T) \rightarrow \mathbb{R}$. Then if $f$ satisfies

$$
\begin{cases}\left(\frac{d}{d t}-\Delta\right) f(\mathbf{p}, t) \leq 0 & \forall(\mathbf{p}, t) \in M^{n} \times[0, T) \text { such that } \nabla f(\mathbf{p})=0 \\ \langle\nabla f, \mu\rangle \leq 0 & \forall(\mathbf{p}, t) \in \partial M^{n} \times[0, T)\end{cases}
$$

then $f(\mathbf{x}, t) \leq \sup _{\mathbf{p} \in M^{n}} f(\mathbf{p}, 0)$ for all $(\mathbf{x}, t) \in M^{n} \times[0, T)$.

Using Lemmas 4.1 and 5.1, we see that we can immediately apply the above to both $F^{2}-2 n t$ and $2 n t-F^{2}$ to give

$$
C_{1}\left(M_{0}\right) \leq F^{2}-2 n t \leq C_{2}\left(M_{0}\right)
$$

Therefore, if our manifold lies between two copies of a hyperbolic solution $\mathbf{G}_{C_{1}}$ and $\mathbf{G}_{C_{2}}$ initially, then it will do so for all time. This also gives the required bounds on $u$, and ensures that $M_{0}$ stays away from the singularity of $\Sigma$ for the entire time a solution exists.

Now using Proposition 4.4 and Lemma 4.3 we consider the evolution of $\frac{H}{S}(C+2 n t)$. We see that

$$
\left(\frac{d}{d t}-\Delta\right) \frac{H}{S}(C+2 n t)=\frac{H}{S}\left(2 n-2(C+2 n t) \frac{H}{S}\right)-2\left\langle\frac{\nabla S}{S}, \nabla \frac{H}{S}(C+2 n t)\right\rangle,
$$

where the final term will disappear at a stationary point. Hence given that $H>0$ on $M_{0}$ and again applying weak maximum principle, we have for $C_{3}, C_{4}>0$

$$
\frac{C_{3}}{C+2 n t} \leq \frac{H}{S} \leq \frac{C_{4}}{C+2 n t}
$$

or for $\widehat{C}_{3}, \widehat{C}_{4}>0$

$$
\widehat{C}_{3} \leq \frac{H}{S} F^{2} \leq \widehat{C}_{4}
$$

If $H \geq 0$ on $M_{0}$ (with equality somewhere), then the constant $C_{3}$ is zero. This estimate implies preservation of weak or strict mean convexity since

$$
H \geq C_{3} \frac{S}{C+2 n t} \geq 0
$$

If we neglect the assumption of initial mean convexity, estimate (4) still holds, although $\widehat{C}_{3} \leq-n$. 
Until now we have not used our assumption, and this is the point at which it comes in, in the form of a sign of the boundary derivative on $H$ (and later $S$ ). Using Proposition 4.4 we get that in the interior of $M$,

$$
\left(\frac{d}{d t}-\Delta\right) H^{2}=-2 H^{2}|A|^{2}-2|\nabla H|^{2},
$$

and from Lemma 5.4 and our assumption, $\nabla_{\mu} H=-2 H^{2} A(\nu, \nu) \leq 0$. By the Weak Maximum Principle we therefore have

$$
H^{2}<C_{5}
$$

Now using Lemmas 4.1, 4.3, 5.1, 5.3 we calculate for $f=S-\frac{\sqrt{C_{5}}}{n} F^{2}$ that

$$
\left(\frac{d}{d t}-\Delta\right) f=2 H-2 \sqrt{C_{5}}-S|A|^{2} \leq-S|A|^{2} \leq 0
$$

and

$$
\langle\nabla f, \mu\rangle=\langle\nabla S, \mu\rangle \leq 0
$$

Again applying the Weak Maximum Principle we see that

$$
S \leq C_{6}\left(M_{0}\right)+\frac{\sqrt{C_{5}}}{n} F^{2}
$$

and hence we get

$$
v>\frac{F^{2}}{\sqrt{1-|\mathbf{x}|^{2}}\left(C_{6}+\frac{\sqrt{C_{5}}}{n} F^{2}\right)}>0 .
$$

We have the estimates required and give the following summary:

Theorem 6.3. Given that $M_{0}$ is spacelike, a solution to equation (1) exists for all time. Mean convexity is preserved by the flow, and if the solution is initially bounded by $\mathbf{G}_{C_{1}}$ and $\mathbf{G}_{C_{2}}$, it will remain so for all time.

Proof. From the gradient estimate above and from our bound $\sqrt{C_{1}+2 n t} \leq u \leq$ $\sqrt{C_{2}+2 n t}$ we have uniform parabolicity of equation (2) and a bound on $|D u|$. Therefore for all time intervals $[0, T]$ from standard results on quasilinear PDEs, for example [9], we have existence of a unique smooth solution. Therefore we have existence of a solution to equation (1) for all time.

\section{IMPROVEMENTS TO ESTIMATES}

We expect our solution to move towards an expanding hyperbolic hyperplane $\mathbf{G}_{k}$, but if this is so the estimates from the previous section are not optimal. We currently have that $F \leq S \leq C_{6}+C_{7} F^{2}$, while on a special solution $S=F$. Also we only have $\frac{C_{3}}{\sqrt{C+2 n t}} \leq H \leq \sqrt{C_{5}}$, while on a special solution we know $H=\frac{n}{F}$. However our ratio of $H$ to $S$, estimate (44), is of the right order.

To improve our estimates we consider

$$
\frac{\left|\nabla F^{2}\right|^{2}}{F^{2}}=4 \frac{\left|F^{\top}\right|^{2}}{F^{2}}=\frac{4}{F^{2}}\langle F-S \nu, F-S \nu\rangle=4 \frac{S^{2}-F^{2}}{F^{2}} .
$$

Note that this quantity is scaling invariant and is zero on our special solution. We show that in fact under the flow it is asymptotically zero. We calculate for 
$J=\frac{S^{2}-F^{2}}{F^{2}}$ that

$$
\begin{aligned}
\left(\frac{d}{d t}-\Delta\right) J=\frac{1}{F^{2}}\left(\frac{d}{d t}-\Delta\right) & \left(S^{2}-F^{2}\right)+\frac{2}{F^{4}}\left\langle\nabla F^{2}, \nabla\left(S^{2}-F^{2}\right)\right\rangle \\
& +\left(S^{2}-F^{2}\right)\left(-\frac{1}{F^{4}}\left(\frac{d}{d t}-\Delta\right) F^{2}-2 \frac{\left|\nabla F^{2}\right|^{2}}{F^{6}}\right) \\
= & \frac{1}{F^{2}}\left[4 H S-2 S^{2}|A|^{2}-2|\nabla S|^{2}-2 n\right. \\
- & \left.2 n J-8 J^{2}+\frac{2}{F^{2}}\left\langle\nabla F^{2}, \nabla\left(S^{2}-F^{2}\right)\right\rangle\right] .
\end{aligned}
$$

Since $|A|^{2} \geq \frac{H^{2}}{n^{2}}$ we estimate

$$
4 S H-2 S^{2}|A|^{2} \leq 2 n^{2}-\frac{2}{n^{2}}\left(S H-n^{2}\right)^{2} \leq 2 n^{2} .
$$

By Cauchy-Schwarz and Young's inequalities we also see that

$$
\begin{aligned}
\frac{1}{F^{2}}\left\langle\nabla F^{2}, \nabla\left(S^{2}-F^{2}\right)\right\rangle-|\nabla S|^{2} & \leq 2 \frac{S}{F} \frac{\left|\nabla F^{2}\right|}{F}|\nabla S|-\frac{\left|\nabla F^{2}\right|^{2}}{F^{2}}-|\nabla S|^{2} \\
& \leq \frac{S^{2}}{F^{2}} \frac{\left|\nabla F^{2}\right|^{2}}{F^{2}}-\frac{\left|\nabla F^{2}\right|^{2}}{F^{2}} \\
& =4 J^{2} .
\end{aligned}
$$

Applying this to the evolution equation for $J$ we have

$$
\left(\frac{d}{d t}-\Delta\right) J \leq \frac{2 n}{F^{2}}[n-1-J]
$$

which implies since the boundary derivative of $J$ is $\langle\nabla J, \mu\rangle \leq 2 \frac{S}{F^{2}}\langle\nabla S, \mu\rangle<0$ that $J$ is bounded by the maximum of its initial value and $n-1$. But we can do better than that. For $C \geq C_{2}$ we see

$$
\begin{aligned}
\left(\frac{d}{d t}-\Delta\right) & J \log (C+2 n t) \\
& \leq \frac{2 n \log (C+2 n t)}{F^{2}}[n-1-J]+\frac{2 n J}{C+2 n t} \\
& \leq \frac{2 n \log (C+2 n t)}{F^{2}}\left[n-1-\left(1-\frac{1}{\log (C+2 n t)}\right) J\right],
\end{aligned}
$$

and by choosing $C$ sufficiently large, for example $C>e^{2}$, then we have the following:

Proposition 7.1. There exist constants $C_{S}, D_{S}, \widetilde{D}_{S}>0$ depending only on $n$ and $M_{0}$ such that

$$
\frac{\left|\nabla F^{2}\right|^{2}}{F^{2}} \leq 4 \frac{C_{S}}{\log \left(D_{S}+2 n t\right)} \leq 4 \frac{C_{S}}{\log \left(\widetilde{D}_{S}+F^{2}\right)}
$$

or equivalently

$$
\frac{S^{2}}{F^{2}} \leq 1+\frac{C_{S}}{\log \left(D_{S}+2 n t\right)} \leq 1+\frac{C_{S}}{\log \left(\widetilde{D}_{S}+F^{2}\right)}
$$


Now the estimate equation (44) implies the following:

Corollary 7.2. There exist constants $C_{1}^{H}$ and $C_{2}^{H}>0$ such that

$$
C_{1}^{H} \leq H F \leq C_{2}^{H}
$$

where $C_{1}^{H}$ is positive if $M_{0}$ is initially mean convex.

Remark 7.3. If we suppose $M_{0}$ is mean convex, we may use the above to calculate the average of $H$ asymptotically in time. From the proof of Lemma 4.1 we have that $\Delta F^{2}=2 H S-2 n$, and therefore we see using Lemma 5.1 and the divergence theorem that

$$
\int_{M} H S d \mu=n \int_{M} d \mu
$$

Therefore since $H>0$ we may estimate

$$
n \leq \frac{\int_{M} H F d \mu}{\int_{M} d \mu} \leq n \sqrt{1+\frac{C_{S}}{\log \left(D_{S}+2 n t\right)}}
$$

which asymptotically corresponds to exactly what we would expect in our special solution.

\section{INTERIOR CURVATURE ESTIMATES}

We obtain some interior estimates on $|A|^{2}$ and its derivatives. Note that on our homothetic solution we get that $|A|^{2}=\frac{n}{F^{2}}$ and we search for estimates of a similar order.

To construct a cutoff function first suppose $\bar{K}: \mathbb{R}_{1}^{n+1} \rightarrow \mathbb{R}$ and define $K$ : $M^{n} \times[0, T) \rightarrow \mathbb{R}$ by $K(x, t)=\bar{K}(\mathbf{F}(x, t))$.

Lemma 8.1. Under $M C F, K$ evolves by

$$
\left(\frac{d}{d t}-\Delta\right) K=-\left.\bar{\nabla}_{\nu} \bar{\nabla}_{\nu} \bar{K}\right|_{\mathbf{F}}-\left.\bar{\Delta} \bar{K}\right|_{\mathbf{F}}
$$

Proof. We see that

$$
\frac{d}{d t} K=H \bar{\nabla}_{\nu} \bar{K}
$$

We also calculate

$$
\Delta K=H \bar{\nabla}_{\nu} \bar{K}+g^{i j} \bar{\nabla}_{\frac{\partial \mathbf{F}}{\partial x^{i}}} \bar{\nabla}_{\frac{\partial \mathbf{F}}{\partial x^{j}}} \bar{K}
$$

using the Weingarten relations. But now by considering locally in a suitable orthonormal coordinate system and noting the sign of $\nu$ we see that

$$
\Delta K=H \bar{\nabla}_{\nu} \bar{K}+\bar{\Delta} \bar{K}+\bar{\nabla}_{\nu} \bar{\nabla}_{\nu} \bar{K},
$$

which gives the lemma.

We now stipulate an additional condition on $\bar{K}$, namely that $\bar{\nabla}_{\mathbf{F}} \bar{K}=0$. That is, the cutoff function is defined on a hyperbolic plane and remains constant on rays from the origin. We define $Y_{\lambda}=\left\{\mathbf{x} \mid\langle\mathbf{x}, \mathbf{x}\rangle=-\lambda^{2}, x_{n+1}>0\right\}$ that is a spacelike embedding of the hyperbolic plane of "radius" $\lambda$. 
Corollary 8.2. Under the condition $\bar{\nabla}_{\mathbf{F}} \bar{K}=0$ we have at $\mathbf{p} \in M$

$$
\begin{aligned}
\left(\frac{d}{d t}-\Delta\right) K & \leq\left|\nabla^{Y_{F}^{2}} \bar{K}\right|^{Y_{F}}(\mathbf{p})\left(\frac{S^{2}}{F^{2}}-1\right)-\Delta^{Y_{F}} \bar{K}(\mathbf{p}) \\
& =\left|\nabla^{Y_{1}^{2}} \bar{K}\right|^{Y_{1}}\left(\frac{\mathbf{p}}{F}\right) \frac{\frac{S^{2}}{F^{2}}-1}{F^{2}}-\frac{1}{F^{2}} \Delta^{Y_{1}} \bar{K}\left(\frac{\mathbf{p}}{F}\right) \\
& \leq \frac{\widetilde{C}_{K}}{F^{2}} .
\end{aligned}
$$

Proof. Since $Y_{F}$ is perpendicular to $\mathbf{F}$ and $\bar{\Delta} \bar{K}$ has no contribution from the $\mathbf{F}$ direction we immediately have $\bar{\Delta} \bar{K}=\Delta^{Y_{F}} \bar{K}$. Similarly we have

$$
\begin{aligned}
\left|\bar{\nabla}_{\nu} \bar{\nabla}_{\nu} \bar{K}\right|_{\mathbf{F}} \mid & =\left|\bar{\nabla}_{\nu-\frac{\langle\nu, \mathbf{F}\rangle}{F^{2}} \mathbf{F}} \bar{\nabla}_{\nu-\frac{\langle\nu, \mathbf{F}\rangle}{F^{2}} \mathbf{F}} \bar{K}\right|_{\mathbf{F}} \mid \\
& =\left|\bar{\nabla}_{\nu+\frac{S}{F^{2}} \mathbf{F}}^{Y_{F}} \bar{\nabla}_{\nu+\frac{S}{F^{2}} \mathbf{F}}^{Y_{F}}\right|_{\mathbf{F}} \mid \\
& \leq \mid \nabla^{Y_{F}} \bar{K}^{Y_{F}}\left(\frac{S^{2}}{F^{2}}-1\right)
\end{aligned}
$$

by Cauchy-Schwarz, giving the first inequality.

The second is using the scaling of $K$ on $Y_{F}$ - this allows us to estimate over $Y_{1}$ rather than $Y_{F}$, where $F$ may vary from point to point. This inequality is simply derived from properties of dilations and the constancy of $\bar{K}$ on rays from $\mathbf{0}$ : Keeping a function constant but dilating the manifold by $\lambda$ while keeping $\bar{K}$ the same, we get that $g_{i j}$ becomes $\lambda^{2} g_{i j}, g^{i j}$ becomes $\lambda^{-2} g^{i j}, \Gamma_{i j}^{k}$ remains $\Gamma_{i j}^{k}$, and so on. This gives the stated formula.

The third of these comes from estimating second derivatives of $\bar{K}$ on $Y_{1}$ and Proposition 7.1 .

The final two conditions we wish $\bar{K}$ to have in addition to that of the above corollary are:

- $0 \leq \bar{K} \leq C$, where $\bar{K}$ restricted to $Y_{1}$ has compact support, and

- $\frac{\left|\nabla^{Y_{1}} K\right|^{2}}{K} \leq \widehat{C}_{K}$ for some $\widehat{C}_{K}>0$.

The question of whether such a function exists is easily solved. For example, if we take the Poincaré model of hyperbolic space (which is isometric to $Y_{1}$ ), we could take $K$ to be the radial function $K(r)=\left(1-E r^{2}\right)_{+}^{3}$. We then calculate in this metric

$$
\frac{\left|\nabla^{\text {Poin }} K\right|^{2}}{K}=\frac{\left(\frac{d K}{d r}\right)^{2}\left(1-r^{2}\right)^{2}}{4 K}=9 E^{2} r^{2}\left(1-E r^{2}\right)_{+}\left(1-E r^{2}\right),
$$

which is clearly bounded (depending on $E$ ) on the unit ball. Furthermore, this function is zero outside a hyperbolic ball and bounded by 1 , and by changing $E$ we may choose the radius of the hyperbolic ball which is $\operatorname{supp}(K)$.

For $K$ satisfying the above we know

$$
\begin{aligned}
|\nabla K|^{2} & =|\bar{\nabla} \bar{K}|^{2}+\left\langle\nabla^{Y_{F}} K, \nu-\frac{S}{F^{2}} \mathbf{F}\right\rangle^{2} \\
& \leq \frac{\widetilde{C}_{S}\left|\nabla^{Y_{1}} K\right|^{2}}{F^{2}}
\end{aligned}
$$


for $\widetilde{C}_{S}=1+\frac{C_{S}}{\log D_{S}}>0$, where we used the Cauchy-Schwarz inequality on the hyperbolic plane and Proposition 7.1. Therefore for such a function we have that at a maximum of $f K$, a point where $f \nabla K+K \nabla f=0$,

$$
\begin{aligned}
\left(\frac{d}{d t}-\Delta\right) f K & \leq \frac{f \widetilde{C}_{K}}{F^{2}}+\widetilde{C}_{S} f \frac{\left|\nabla^{Y_{1}} K\right|^{2}}{F^{2} K}+K\left(\frac{d}{d t}-\Delta\right) f \\
& \leq \frac{f C_{K}}{F^{2}}+K\left(\frac{d}{d t}-\Delta\right) f
\end{aligned}
$$

where $C_{K}>0$.

Lemma 8.3. Let $L \subset \mathbb{R}_{1}^{n+1}$ be such that if $\mathbf{x} \in L$, then $\lambda x \in L \quad \forall \lambda \in \mathbb{R}$, and so that $Y_{1} \cap L$ is a compact set of minimum hyperbolic distance $d>0$ from $\Sigma$ with a smooth boundary. Then on $M_{t} \cap L$

$$
|A|^{2} \leq \frac{C_{A}}{F^{2}}
$$

where the constant depends on $d$, the second derivatives of the boundary of $Y_{1} \cap L$, $n$ and $M_{0}$.

Proof. Since we have suitable cutoff functions (if necessary just using the radial one with sufficiently large $E$ ), the proof comes down to evolution equations. We calculate that for $f_{0}=|A|^{2}(D+2 n t)$,

$$
\begin{aligned}
\left(\frac{d}{d t}-\Delta\right) f_{0} & =2 n|A|^{2}-2(D+2 n t)\left(|A|^{4}+|\nabla A|^{2}\right) \\
& \leq \frac{2 n f_{0}}{D+2 n t}-(D+2 n t)|A|^{4} .
\end{aligned}
$$

Now applying equation (5) we have by choosing $D$ large enough that

$$
\left(\frac{d}{d t}-\Delta\right) K f_{0} \leq \frac{f_{0}}{F^{2}}\left[C_{K}+2 n K-B f_{0} K\right]
$$

for some $B>0$ depending on $C_{1}$ and $C_{2}$ (see equation (3) ). Therefore since $K=0$ at the boundary we have the lemma.

Lemma 8.4. For $L$ as in the previous lemma we have that for all $m \geq 1$ there exists a constant $C_{A, m}$ depending on $m, n, L, d, M_{0}$ and the second derivatives of the boundary of $Y_{1} \cap L$ such that

$$
\left|\nabla^{m} A\right|^{2} \leq \frac{C_{A, m}}{F^{2}}
$$

Proof. The proof is by induction. Writing $J_{1}=|A|^{2}(D+2 n t)+E<C_{A}+E$ where $E>0$ is a constant yet to be chosen, we define

$$
f_{1}=(D+2 n t)|\nabla A|^{2} J_{1} .
$$


Using Proposition 4.4, the Cauchy-Schwarz inequality and the above lemma, writing $C_{n}$ for any positive constant depending only on $n, K$ and $M_{0}$ we have

$$
\begin{aligned}
\left(\frac{d}{d t}-\Delta\right) f_{1} \leq(D+2 n t)[ & -2 J_{1}\left|\nabla^{2} A\right|^{2}-2(D+2 n t)|\nabla A|^{4} \\
& \left.+\frac{C_{n}(E+1)}{D+2 n t}|\nabla A|^{2}+8(D+2 n t)\left|\nabla^{2} A\right||\nabla A|^{2}|A|\right] \\
\leq(D+2 n t)\left[\frac{4|\nabla A|^{4}|A|^{2}(D+2 n t)^{2}}{\left(|A|^{2}(D+2 n t)+E\right)^{2}}-2(D+2 n t)|\nabla A|^{4}\right. & \left.+\frac{C_{n}(E+1)}{D+2 n t}|\nabla A|^{2}\right] \\
\leq(D+2 n t)\left[\frac{C_{n}}{E^{2}}|\nabla A|^{4}(D+2 n t)-2(D+2 n t)|\nabla A|^{4}\right. & \left.+\frac{C_{n}(E+1)}{D+2 n t}|\nabla A|^{2}\right] .
\end{aligned}
$$

We now choose $E$ sufficiently large such that the coefficient $\frac{C_{n}}{E^{2}} \leq \frac{1}{2}$, and therefore

$$
\begin{aligned}
\left(\frac{d}{d t}-\Delta\right) f_{1} & \leq(D+2 n t)\left[-\frac{3}{2}(D+2 n t)|\nabla A|^{4}+\frac{C_{n}}{D+2 n t}|\nabla A|^{2}\right] \\
& \leq \frac{C_{n} f_{1}}{D+2 n t}-C_{n} f_{1}^{2}
\end{aligned}
$$

where here again we used the bound on $J_{1}$. Substituting into equation (5) we see that

$$
\left(\frac{d}{d t}-\Delta\right) f_{1} K \leq f_{1}\left(\frac{C_{n}}{F^{2}}-C_{n} f_{1}\right)
$$

In fact the same argument holds for

$$
f_{m}=(B+2 n t)\left|\nabla^{m} A\right|^{2}\left(\left|\nabla^{m-1} A\right|^{2}(B+2 n t)+D\right)
$$

by induction. This follows from:

- The fact that we don't use the extra negative term, $-|A|^{4}$, from the evolution equation of $|A|^{2}$.

- The extra positive term from the evolution equation of $\left|\nabla^{m-1} A\right|^{2}$ is

$$
\sum_{i+j+k=m-1} \nabla^{i} A * \nabla^{j} A * \nabla^{k} A * \nabla^{m-1} A \leq \frac{C}{(B+2 n t)^{2}}
$$

by the inductive hypothesis, which is of the right order for the above equations to still hold.

- The only other remaining difference is an extra term in the estimate on the evolution equation of $\left|\nabla^{m} A\right|^{2}$ which becomes a positive term $C_{n} \frac{\sqrt{f_{m}}}{D+2 n t}$ in the evolution of $f_{m}$. This is easily dealt with by using $-\epsilon f_{m}^{2}$.

Hence an identical argument to the above gives the lemma.

\section{Convergence And Renormalisation}

In this section our purpose is to define the shape of the solution as $t \rightarrow \infty$. For this some notion of blowdown will be needed. 
Definition 9.1. If $\mathbf{F}: M^{n} \times[0, \infty) \rightarrow \mathbb{R}_{1}^{n+1}$ satisfies equation (1), then let $\widehat{\mathbf{F}}=$ $\psi(t) \mathbf{F}$ where $\psi(t)$ is some factor such that the area of $\widehat{\mathbf{F}}(M)$ is 1 . For any geometric quantity $f$ on $\mathbf{F}$ we will denote the same quantity $\widehat{f}$ on $\widehat{\mathbf{F}}$.

For $\mathbf{G}: M^{n} \times[0, \infty) \rightarrow \mathbb{R}_{1}^{n+1}$ we will say $\mathbf{F} \rightarrow \mathbf{G}$ as $t \rightarrow \infty$ in $C^{0}, C^{1}, \ldots$ if $\widehat{\mathbf{F}} \rightarrow \widehat{\mathbf{G}}$ as $t \rightarrow \infty$ in $C^{0}, C^{1}, \ldots$

Remark 9.2. It is usual (as in [7]) to renormalise time as well. Indeed we may also do so here, by defining $s=\int_{0}^{t} \psi(r)^{2} d r$. We then obtain

$$
\frac{d \widehat{\mathbf{F}}}{d s}=\frac{d \widehat{\mathbf{F}}}{d t} \frac{d t}{d s}=\psi^{-2}\left(\psi H \nu-\psi^{-1} \frac{1}{n} \frac{\int_{M} H^{2} d \mu}{\int_{M} d \mu} \mathbf{F}\right)=\widehat{H} \nu+\frac{\widehat{\mathbf{F}}}{n} \int_{\widehat{M}} \widehat{H}^{2} d \widehat{\mu} .
$$

In actual fact Lemma 9.3 will show that $s \geq C \log (D+2 n t)$, and hence we need not make a distinction between $s \rightarrow \infty$ and $t \rightarrow \infty$.

It will be useful to have estimates on the quantity $\psi$.

Lemma 9.3. There exist constants $C_{Y}, \widetilde{C}_{Y}>0$ such that for time $t$ sufficiently large,

$$
\frac{C_{Y}}{F} \leq \psi(t) \leq C_{Y} \frac{\sqrt{1+\frac{C_{S}}{\log \left(\widetilde{D}_{S}+F^{2}\right)}}}{F} \leq \frac{\widetilde{C}_{Y}}{F} .
$$

Proof. Let $\mathbf{Y}$ be a parametrisation of $Y_{1}$. Then any spacelike manifold contained within the lightcone may be written as $\mathbf{Z}=u(x) \mathbf{Y}(x)$. By standard methods we get the following identities:

$$
\begin{aligned}
g_{i j}^{Z} & =u^{2} g_{i j}^{Y}-D_{i} u D_{j} u, \\
g_{Z}^{i j} & =\frac{1}{u^{2}}\left(g_{Y}^{j k}+\frac{D_{a} u g_{Y}^{a j} D_{b} u g_{Y}^{b k}}{u^{2}-\left|\nabla^{Y} u\right|^{2}}\right), \\
\operatorname{det}\left(g_{i j}^{Z}\right) & =\left(u^{2}\right)^{n-1}\left(u^{2}-\left|\nabla^{Y} u\right|^{2}\right) \operatorname{det} g_{i j}^{Y} .
\end{aligned}
$$

From these we may deduce that

$$
\left|\nabla^{Y} u\right|^{2}=\frac{u^{2}\left|\nabla u^{2}\right|^{2}}{4 u^{2}+\left|\nabla u^{2}\right|^{2}}
$$

Since $u=F$ and we may write the area of $M$ as the integral over the interior of $\Sigma$ intersected with $Y_{1}$, we call this set $\mathcal{B}$. Therefore

$$
\begin{aligned}
\int_{M} d \mu & =\int_{\mathcal{B}} u^{n} \sqrt{1-\frac{\left|\nabla^{Y} u\right|^{2}}{u^{2}}} d \mu_{Y_{1}} \\
& =\int_{\mathcal{B}} F^{n} \sqrt{\frac{1}{1+\frac{\left|\nabla F^{2}\right|^{2}}{4 F^{2}}}} d \mu_{Y_{1}},
\end{aligned}
$$

and so by using Proposition [7.1 and equation (3) we see for $t$ large enough there exists a $C_{\mathcal{B}}$ such that

$$
C_{\mathcal{B}} F^{n} \sqrt{\frac{1}{1+\frac{C_{S}}{\log \left(\widetilde{D}_{S}+F^{2}\right)}}} \leq \int_{M} d \mu \leq C_{\mathcal{B}} F^{n} .
$$

Since $\psi=\left(\int_{M} d \mu\right)^{-\frac{1}{n}}$ we have the lemma. 
Theorem 9.4. Any initially spacelike solution of equation (1) with a convex cone boundary condition will converge as time tends towards infinity to some $\mathbf{G}_{k}$ in the $C^{1}$ norm. Furthermore, there exists an increasing sequence of $t_{i} \rightarrow \infty$ such that the $\widehat{M}_{t_{i}}$ converge to the solution in the interior in the $C^{\infty}$ topology.

Proof. Under a dilation by a factor $\lambda, \mathcal{D}_{\lambda}$ we have $\mathcal{D}_{\lambda}\left(F^{2}\right)=\lambda^{2} F^{2}, \mathcal{D}_{\lambda} S=\lambda S$, and so on. Hence from equation (3), Proposition [7.1. Corollary 7.2 and the above estimates on the dilation factor we get

$$
\begin{aligned}
\widehat{C}_{1} & \leq \widehat{F}^{2} \leq \widehat{C}_{2}, \\
0 & \leq\left|\nabla \widehat{F}^{2}\right|^{2} \leq \frac{\widehat{C}_{S}}{\log \left(\widetilde{D}_{S}+F^{2}\right)}, \\
\widehat{C}_{1}^{H} \leq \widehat{H} & \leq \widehat{C}_{2}^{H},
\end{aligned}
$$

where $\widehat{C}_{1}, \widehat{C}_{2}, \widehat{C}_{S}, \widehat{C}_{2}^{H}>0$ and $\widehat{C}_{1}^{H}>0$ if $C_{1}^{H}>0$, and all of these constants depend only on $n$ and $M_{0}$. The first of these is boundedness of the renormalised hypersurface and the second implies that we have in fact $C^{1}$ convergence to a hypersurface with $\left|\nabla F^{2}\right|=0$. Therefore these estimates imply $C_{1}$ convergence of $\widehat{M}$ to $Y_{R}$, the hyperbolic hyperplane of radius

$$
R=\left(\int_{\mathcal{B}} d \mu_{Y_{1}}\right)^{-\frac{1}{n}}
$$

where $\mathcal{B}$ is as in the previous lemma.

By Lemmas 8.3 and 8.4 we have that for $L$ as in Lemma 8.3 , then for all time on $M_{t} \cap L$

$$
\begin{aligned}
\max \left\{\frac{\widehat{C}_{1}^{H}}{n}, 0\right\}^{2} & \leq|\widehat{A}|^{2} \leq \widehat{C}_{A}, \\
0 & \leq\left|\nabla^{m} \widehat{A}\right|^{2} \leq \widehat{C}_{A, m},
\end{aligned}
$$

where the constant depends on the boundary of $L$ and how far $L$ is from $\Sigma$.

Now using Arzelá-Ascoli repeatedly, a standard diagonal argument completes the theorem.

\section{ACKNOWLEDGEMENT}

This paper is part of the author's Ph.D. thesis. The author would like to thank his supervisor, W. Klingenberg, for his support, enthusiasm and excellent advice.

\section{REFERENCES}

[1] Steven J. Altschuler and Lang F. Wu, Translating surfaces of the non-parametric mean curvature flow with prescribed contact angle, Calc. Var. Partial Differential Equations 2 (1994), no. 1, 101-111, DOI 10.1007/BF01234317. MR1384396 (97b:58032)

[2] John A. Buckland, Mean curvature flow with free boundary on smooth hypersurfaces, J. Reine Angew. Math. 586 (2005), 71-90, DOI 10.1515/crll.2005.2005.586.71. MR2180601 (2006h:53058)

[3] Klaus Ecker, Interior estimates and longtime solutions for mean curvature flow of noncompact spacelike hypersurfaces in Minkowski space, J. Differential Geom. 46 (1997), no. 3, 481-498. MR 1484889 (98j:58034)

[4] Klaus Ecker, Mean curvature flow of spacelike hypersurfaces near null initial data, Comm. Anal. Geom. 11 (2003), no. 2, 181-205. MR2014875 (2004k:53098)

[5] Klaus Ecker and Gerhard Huisken, Parabolic methods for the construction of spacelike slices of prescribed mean curvature in cosmological spacetimes, Comm. Math. Phys. 135 (1991), no. 3, 595-613. MR1091580 (92g:53058) 
[6] Brendan Guilfoyle and Wilhelm Klingenberg. Proof of the Carathéodory conjecture. Ar $\chi$ iv preprint, 2012. http://arxiv.org/abs/0808.0851.

[7] Gerhard Huisken, Flow by mean curvature of convex surfaces into spheres, J. Differential Geom. 20 (1984), no. 1, 237-266. MR772132 (86j:53097)

[8] Gerhard Huisken, Nonparametric mean curvature evolution with boundary conditions, J. Differential Equations 77 (1989), no. 2, 369-378, DOI 10.1016/0022-0396(89)90149-6. MR.983300 (90g:35050)

[9] Gary M. Lieberman, Second order parabolic differential equations, World Scientific Publishing Co. Inc., River Edge, NJ, 1996. MR 1465184 (98k:35003)

[10] Axel Stahl, Convergence of solutions to the mean curvature flow with a Neumann boundary condition, Calc. Var. Partial Differential Equations 4 (1996), no. 5, 421-441, DOI 10.1007/BF01246150. MR1402731 (97f:58043)

[11] Axel Stahl, Regularity estimates for solutions to the mean curvature flow with a Neumann boundary condition, Calc. Var. Partial Differential Equations 4 (1996), no. 4, 385-407, DOI 10.1007/BF01190825. MR1393271 (97h:53002)

Department of Mathematics, University of Konstanz, Zukunftskolleg, Box 216, 78457 KOnStanZ, GERMAnY

E-mail address: benjamin.lambert@uni-konstanz.de 